\title{
A Novel Two-dimensional Quantitative Shear Wave Elastography Mode for Differential Diagnosis of Benign and Malignant Thyroid Nodules
}

\author{
Ying Zhang, MD ${ }^{a, b, c}$, Yifeng Zhang, MD ${ }^{a, b, c, *}$, Hanxiang Wang, MD ${ }^{a, b, c}$, Anqi Zhu, MD ${ }^{a, b, c}$, Hui Shi, MD ${ }^{a, b, c}$ \\ ${ }^{a}$ Department of Medical Ultrasound, Shanghai Tenth People's Hospital, Ultrasound Research and Education Institute, Tongji University \\ Cancer Center, ${ }^{b}$ Shanghai Engineering Research Center of Ultrasound Diagnosis and Treatment, ${ }^{c}$ Tongji University School of Medicine, \\ Shanghai, China \\ Received November 01, 2019; revision received January 28, 2020; accepted February 02, 2020
}

\begin{abstract}
Background: To evaluate the diagnostic performance of a novel two-dimensional quantitative shear wave elastography (SWE) of virtual touch tissue imaging quantification (VTIQ) in the differential diagnosis between benign and malignant thyroid nodules.
\end{abstract}

Methods: Two hundred and six solid thyroid nodules were included in this prospective study. Conventional ultrasound and VTIQ examinations were performed. The diagnostic performances of US, VTIQ, and the combination of the two methods were compared. The usefulness of VTIQ in different size of nodules was also assessed.

Results: There were 113 benign nodules and 93 malignant nodules on fine needle aspiration (FNA) or histology. The shear wave velocities (SWVs) in malignant nodules were all significantly higher than those in benign nodules (SWV max: $4.01 \pm$ $1.72 \mathrm{~m} / \mathrm{s}$ vs $3.25 \pm 1.01 \mathrm{~m} / \mathrm{s}$, SWV min: $2.87 \pm 0.69 \mathrm{~m} / \mathrm{s}$ vs $2.46 \pm 0.67 \mathrm{~m} / \mathrm{s}$, SWV mean: $3.33 \pm 0.89 \mathrm{~m} / \mathrm{s}$ vs $2.85 \pm 0.81 \mathrm{~m} / \mathrm{s}$, SWV median: $3.31 \pm 0.88 \mathrm{~m} / \mathrm{s}$ vs $2.82 \pm 0.77 \mathrm{~m} / \mathrm{s}$, all P value $<0.001)$. After combined VTIQ with ultrasound, the diagnostic performances improved compared with ultrasound alone or VTIQ alone. As for lesions of different sizes, areas under curve (AUC) for the largest diameter of nodules $\leqslant 1.0 \mathrm{~cm}$ was 0.711 , which was lower than that for the largest diameter of the nodules > $1.0 \mathrm{~cm}$ (AUC was 0.862$)(P<0.05)$. Conventional ultrasound features such as halo sign and microcalcification were predictors for lymphatic metastasis in malignant thyroid nodules $(P<0.05)$.

Conclusions: VTIQ is a new method for measuring stiffness of thyroid lesions qualitatively. The diagnostic performance of combined using VTIQ and conventional ultrasound was better than that of using either alone. Malignant thyroid nodules with conventional ultrasound features such as halo sign and microcalcification were more likely to have lymphatic metastasis. The evaluation of small thyroid lesions remained a difficult problem.

Key words: Ultrasound; Elastography; Shear wave; VTIQ; ARFI Imaging; Thyroid nodule

Advanced Ultrasound in Diagnosis and Therapy 2020;03:195-203

DOI: $10.37015 / A U D T .2020 .190039$

onventional ultrasound images reveal differences in the acoustic properties of soft tissues (mainly echogenicity), whereas ultrasound-based elasticity images are able to reveal the differences in the elastic properties of soft tissues [1]. The benefit of elasticity imaging bases on the fact that many soft tissues can share similar ultrasonic echogenicity but may have different mechanical properties can be used to clearly

\footnotetext{
* Corresponding Author: Department of Medical Ultrasound, Shanghai Tenth People's Hospital, Ultrasound Research and Education Institute, Tongji University School of Medicine, Shanghai Engineering Research Center of Ultrasound Diagnosis and Treatment, No. 301, Yanchangzhong Road, Shanghai, China.

e-mail:27763289@qq.com
} unrestricted use, distribution and reproduction in any medium provided that the original work is properly attributed. 
visualize normal anatomy and show pathological lesions [2]. Ultrasound elastography has been developed to study the hardness/elasticity of thyroid nodules and then differentiate malignant nodules from benign ones. It has also been proven to be an adjunctive tool of conventional ultrasound [3].

There are two kinds of elastography (strain and shear wave elastography) that are currently used in clinical practice. Strain elastography bases on the measurement of the tissue deformation generated by applying pressure, including manual compression and acoustic radiation force impulse (ARFI) excitation. Strain imaging is essentially qualitative or semi-quantitative method [4]. The sensitivity, specificity, positive predictive value (PPV), and negative predictive value (NPV) of combined use of ultrasound and strain elastography for diagnosing thyroid malignancy were reported to be $41-98 \%, 34-$ $94.8 \%, 22.3-86.3 \%$ and $83-100 \%$ respectively. The varied diagnostic ability of strain elastography might due to the individual experience of the operator and the lack of quantitative information about the tissue elasticity although semi-quantitative evaluation was applied in some studies [5].

In contrast to strain elastography, shear wave elastography (SWE) provides quantitative elastic information on the basis of the shear waves orthogonal to the direction of the tissue displacement. Until recently, the most widely used SWE is induced by acoustic radiation force impulse (ARFI). It includes two methods for the clinical evaluation of thyroid nodules: point shear wave speed measurement (e.g. Virtual TouchTM Quantification, VTQ) and two-dimensional shear wave elastography (2D-SWE) (e.g. Shear WaveTM Elastography, SWETM). As SWE depends on the production of radiation force by the transducer, it is theoretically more operator-independent and reproducible. Many studies of VTQ for ARFI and SWE have proved that they were valuable adjunct to gray-scale ultrasound in improving the diagnostic performance for predicting thyroid malignancy $[6,7]$.

Virtual touch imaging quantification (VTIQ) is a novel form of 2D-SWE imaging and provides the advantages of both quantitative and relative stiffness imaging combined in one display [8]. Unlike VTQ which provides only single point shear wave velocity (SWV), VTIQ synthesizes information from up to 256 sequential acquisition beam lines inside a two-dimensional user defined region of interest (ROI) to display a qualitative and quantitative map of SWVs ranging between 0.5 and $10 \mathrm{~m} / \mathrm{s}$, as well as qualitative maps for shear wave quality, travel time, and tissue displacement. The purposes of this study were to evaluate the usefulness of VTIQ for predicting malignant thyroid lesion and to compare the diagnostic performance of VTIQ with conventional ultrasound for differentiating benign and malignant thyroid nodules.

\section{Materials and Methods}

\section{Patients}

From June 2014 to March 2019, seven hundred and twelve solid thyroid nodules were imaged with conventional ultrasound and VTIQ. Written informed consent was obtained from all patients and the study was approved by the Ethical Committee of Shanghai Tenth People's Hospital. The inclusion criteria of this prospective study were as follows: (1) solid or almost solid thyroid nodules detected by ultrasound examination; (2) no previous treatment performed on thyroid; (3) age $\geqslant 18$ years. Five hundred and six thyroid nodules were excluded for the following reasons: nodules were lack of FNA or pathological confirmation $(n=461)$; nodules cannot be diagnosed by FNA without pathological confirmation $(n=19)$; imaging data of VTIQ was not complete $(n=12)$; the quality map of SWE examination showed poor $(n=14)$. The flowchart of the patient selection is shown in Fig 1. Finally, two hundred and six solid thyroid nodules in 193 patients (mean age, $49.3 \pm 12.9$ years; range, $24-81$ years) were included in this prospective study. Patients included 154 women (mean age, $49.3 \pm 12.4$ years) and 39 men (mean age, $49.1 \pm 15.0$ years). The largest diameter of the nodules ranged from $0.4 \mathrm{~cm}$ to $5.8 \mathrm{~cm}$ (mean value, $12.3 \pm 9.9 \mathrm{~mm}$ ).

\section{Conventional ultrasound}

Conventional ultrasound and VTIQ examinations were performed using the Siemens S3000 ultrasound machine (Siemens Medical Solutions, Mountain View, CA, USA) equipped with a 9L4 linear array transducer (transducer frequency range, 4-9 MHz). Conventional ultrasound and VTIQ examinations were performed by one radiologist with more than 7 years' experience in thyroid ultrasound.

For conventional ultrasound, evaluations of the following ultrasound features were performed on all thyroid nodules: echogenicity (mixed-, hyper-, iso, hypo-, or marked hypo-echogenicity, with reference to adjacent thyroid parenchyma or strap muscle), halo sign, spot microcalcifications (hyperechoic spots less than $2 \mathrm{~mm}$, without acoustic shadowing), shape (ovoid to round, taller than wide, or irregular), margin (well-defined or ill-defined), and lymphatic findings (metastasis or no metastasis). Color-flow Doppler US was also performed to evaluate the vascularity in lesion (negative, central, peripheral or mixed vascularity-both central and peripheral vascularity in one lesion). The 
data was prospectively recorded in the ultrasound reports before fine needle aspiration (FNA) and surgery. When thyroid nodules showed one or more of these suspicious ultrasound features, they were regarded as suspicious for malignancy, and the other nodules that had no suspicious ultrasound features were regarded as probably benign.

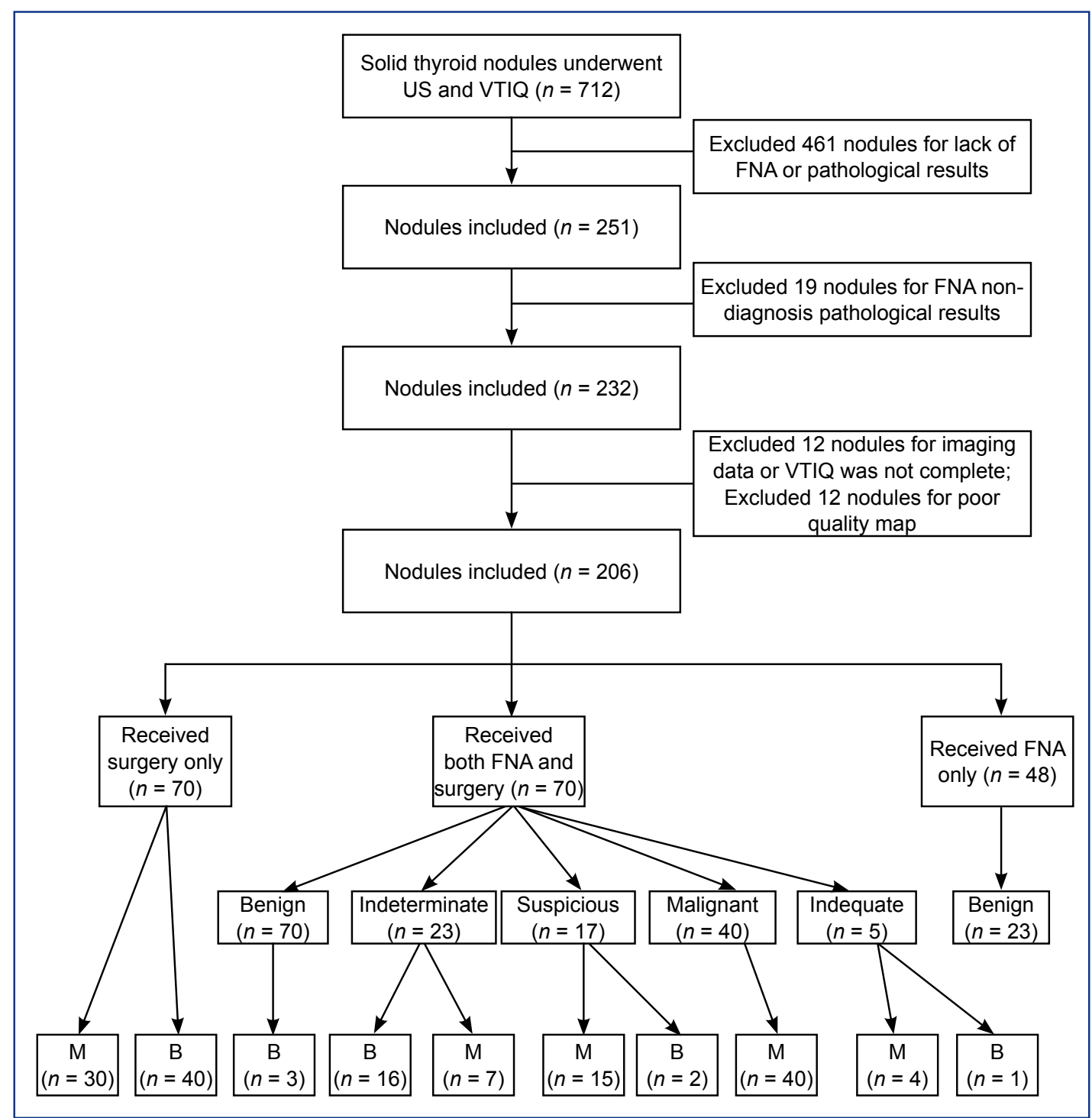

Figure 1 Flowchart of patients' selection in this study.

Table 1 The various SWV values in nodules with different pathological types $(\mathrm{m} / \mathrm{s})$

\begin{tabular}{lcccc}
\hline \multicolumn{1}{c}{ Item } & SWVmax & SWVmin & SWVmean & SWVmedian \\
\hline Nodular goiter & $3.05 \pm 0.69$ & $2.42 \pm 0.57$ & $2.70 \pm 0.55$ & $2.68 \pm 0.56$ \\
Adenoma & $2.77 \pm 0.51$ & $1.69 \pm 0.33$ & $2.22 \pm 0.33$ & $2.21 \pm 0.27$ \\
Hashimoto's nodule & $2.99 \pm 0.79$ & $2.35 \pm 0.59$ & $2.64 \pm 0.66$ & $2.66 \pm 0.68$ \\
Papillary thyroid carcinoma & $4.08 \pm 1.69$ & $2.92 \pm 0.66$ & $3.39 \pm 0.83$ & $3.37 \pm 0.83$ \\
\hline
\end{tabular}

SWV, shear wave velocity

\section{VTIQ}

VTIQ was performed after conventional ultrasound examination. VTIQ function (SIEMENS Medical Solutions, Mountain View, CA, USA) was built in the ultrasound machine. When VTIQ elastography was performed, the transducer was placed on the neck with light pressure and a user-defined ROI (maximum size: $25 \mathrm{~mm}$ to $38 \mathrm{~mm}$ ) was placed and acoustic push pulses 
were applied across the ROI. The ROI box was adjusted to include the nodule (occupying about $70-80 \%$ of the whole ROI) with adequate surrounding thyroid tissue (occupying about $20-30 \%$ of the whole ROI). If the dimension of the nodule exceeded the limit of the ROI, the ROI was placed on the connection between the nodule and the surrounding thyroid tissue; thus, the ROI included both a part of nodule and some surrounding thyroid tissue. VTIQ elastography measurement was initiated in the longitudinal section of the thyroid lesion. VTIQ provides four imaging modes: 1) Velocity; 2) Quality; 3) Travel Time; 4) Displacement. The four modes display the features in the ROI with a color scale as follows: Velocity maps from red (indicates high SWV), yellow or green (indicates intermediate SWV) to blue (indicates low SWV); Quality maps from green (indicates high quality), yellow (indicates intermediate quality) to red (indicates low quality); Travel Time maps from red (indicates fast) to blue (indicates slow); Displacement maps from dark blue (indicates low displacement) to light blue (indicates high displacement). The shear wave quality mode is useful for interpreting whether the shear wave is of sufficient magnitude with adequate signal to noise ratio (SNR) to accurately estimate SWV in the SWV display. Then the imaging mode was shifted to shear wave velocity mode after shear wave quality mode. Both quantitative result and relative stiffness imaging can be shown in the ROI. The SWV can be quantitatively measured in meters per second $(\mathrm{m} / \mathrm{s})$ within the ROI, up to $10 \mathrm{~m} / \mathrm{s}$, corresponding to $300 \mathrm{kPa}$ in Young's modulus. To ensure effective SWV measurement, the sample frame should be placed on the sites corresponding to the green areas on shear wave quality mode and the yellow or red areas on shear wave quality mode should be avoided. And at the same time, the sample frame should be placed on the solid portion of the nodule and calcified portions of the nodule must be avoided. Shear wave velocity mode provides both quantitative and relative stiffness imaging combined in one display. In general, 5 to 7 measurements on the color map within the ROI including the high SWV area (red area) and low SWV area (blue area) were performed (Fig 2 and Fig 3). The highest, lowest, median, and mean SWV values were documented or calculated for further analysis. To investigate whether the nodule size might affect the diagnostic performance of VTIQ on thyroid nodules, the nodules were divided into two groups: Group 1: the largest diameter of nodule $\leqslant 1.0 \mathrm{~cm}$; Group 2: the largest diameter of nodule $>1.0 \mathrm{~cm}$.
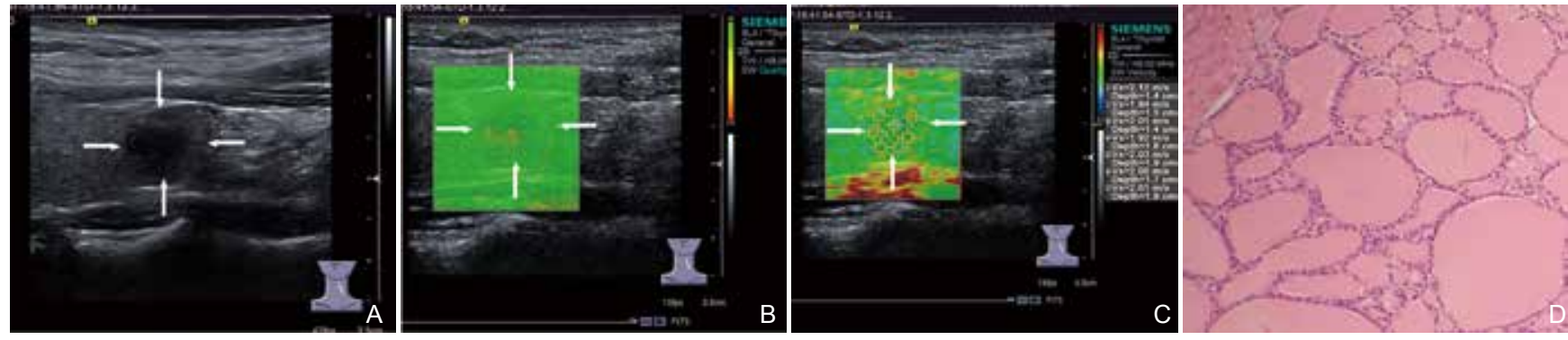

Figure 2 A 40-year-old female with nodular goiter. 2A: Conventional ultrasound shows marked hypo echogenicity and ill-defined margin of the nodule (arrows). 2B: shear wave (SW) Quality map shows almost green in the nodule (arrows), indicating high quality image. 2C: SW velocity map shows both quantitative and relative stiffness imaging in the region of interest (ROI). The red area is corresponding to the highest shear wave velocity (SWV) $2.12 \mathrm{~m} / \mathrm{s}$. The blue area is corresponding to the lowest SWV $1.84 \mathrm{~m} / \mathrm{s}$. The median value is $2.01 \mathrm{~m} / \mathrm{s}$; and the mean SWV is calculated to be $1.99 \mathrm{~m} / \mathrm{s}$. 2D: Histology confirms the diagnosis of nodular goiter. Hematoxylin and eosin stain.
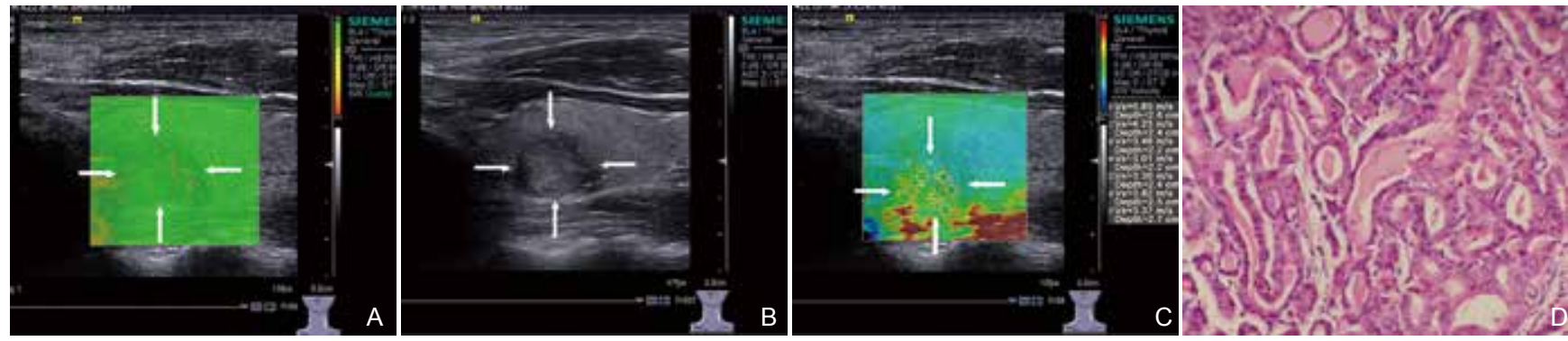

Figure 3 A 32-year-old male with papillary thyroid carcinoma. 3A: Conventional ultrasound shows iso echogenicity, ovoid to round, and ill-defined margin of the nodule (arrows). 3B: SW Quality map shows almost green in the nodule (arrows), indicating high quality image. 3C: SW Velocity map shows both quantitative and relative stiffness imaging in the ROI. The red area is corresponding to the highest SWV of 5.65 m/s. The blue area is corresponding to the lowest SWV of $3.01 \mathrm{~m} / \mathrm{s}$. The median value is $3.46 \mathrm{~m} / \mathrm{s}$; and the mean SWV is calculated to be $3.82 \mathrm{~m} / \mathrm{s}$. $3 \mathrm{D}$ : Histology confirms the diagnosis of papillary thyroid carcinoma. Hematoxylin and eosin stain. 
Zhang et al. SWV for differential diagnosis of thyroid nodules

Table 2 Comparison of various elasticity indices values of VTIQ and their AUC between benign and malignant thyroid nodules

\begin{tabular}{|c|c|c|c|c|c|c|c|}
\hline \multirow{2}{*}{ Item } & \multicolumn{3}{|c|}{ Pathological results } & \multicolumn{4}{|c|}{ Diagnostic performance } \\
\hline & Benign & Malignant & $P$ value & Cut-off value & AUC & $(95 \% \mathrm{CI})$ & $P$ value \\
\hline $\mathrm{SWV}_{\max }(\mathrm{m} / \mathrm{s})$ & $3.25 \pm 1.01$ & $4.01 \pm 1.72$ & $<0.001$ & $3.73 \mathrm{~m} / \mathrm{s}$ & 0.727 & $(0.660-0.786)$ & $<0.001$ \\
\hline $\mathrm{SWV}_{\min }(\mathrm{m} / \mathrm{s})$ & $2.46 \pm 0.67$ & $2.87 \pm 0.69$ & $<0.001$ & $2.50 \mathrm{~m} / \mathrm{s}$ & 0.750 & $(0.685-0.807)$ & $<0.001$ \\
\hline $\mathrm{SWV}_{\text {mean }}(\mathrm{m} / \mathrm{s})$ & $2.85 \pm 0.81$ & $3.33 \pm 0.89$ & $<0.001$ & $2.99 \mathrm{~m} / \mathrm{s}$ & 0.753 & $(0.688-0.810)$ & $<0.001$ \\
\hline $\mathrm{SWV}_{\text {median }}(\mathrm{m} / \mathrm{s})$ & $2.82 \pm 0.77$ & $3.31 \pm 0.88$ & $<0.001$ & $3.01 \mathrm{~m} / \mathrm{s}$ & 0.760 & $(0.696-0.817)$ & $<0.001$ \\
\hline
\end{tabular}

Data are means \pm standard deviations, with ranges in parentheses. VTIQ, virtual touch imaging quantification; SWV, shear wave velocity; AUC, area under the ROC curve; $95 \% \mathrm{CI}, 95 \%$ confidence interval
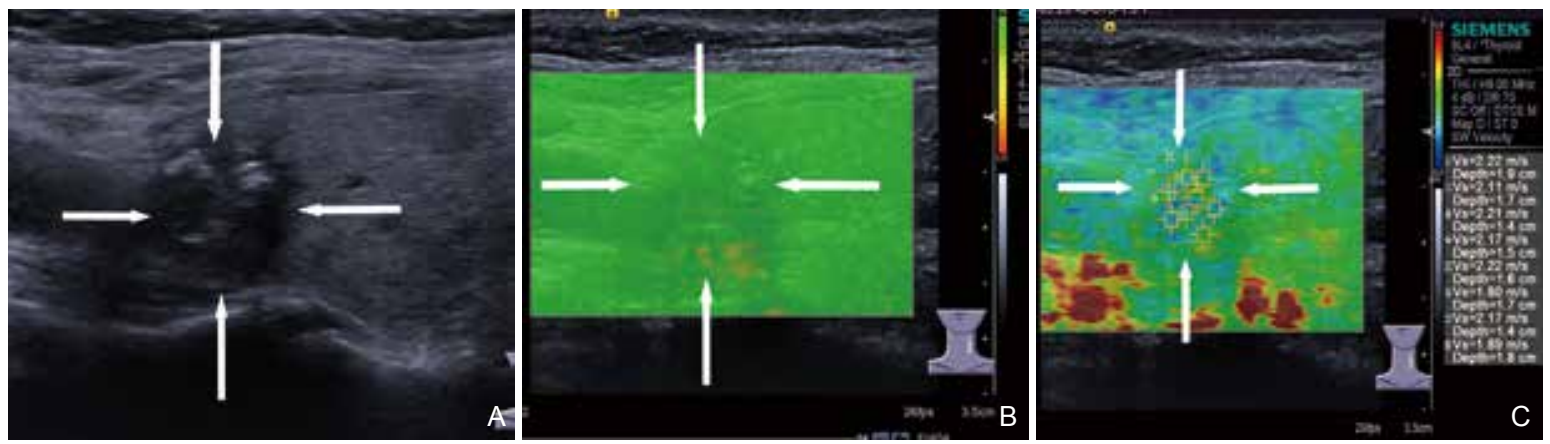

Figure 4 A 57-year-old female with papillary thyroid carcinoma. 4A: Conventional ultrasound shows the nodule with hypo echogenicity, ill-defined margin and some macrocalcifications (arrows). 4B: shear wave (SW) Quality map shows yellow and green in the nodule (arrows), indicating poor quality image and cannot be adopted. 4C: velocity map shows both quantitative and relative stiffness imaging in the region of interest (ROI). The highest shear wave velocity (SWV) is $2.22 \mathrm{~m} / \mathrm{s}$. The lowest SWV is $1.80 \mathrm{~m} / \mathrm{s}$. The median value is $2.17 \mathrm{~m} / \mathrm{s}$; and the mean SWV is calculated to be $2.13 \mathrm{~m} / \mathrm{s}$.

\section{Statistical Analysis}

Quantitative data was expressed as the mean \pm standard deviation. The mean SWV values of benign and malignant nodules were compared by t statistics. The ratios of suspicious malignancy ultrasound features between benign and malignant thyroid nodules were compared by $\chi 2$ statistics. If there were cells with fewer than 5 observations, the Fisher exact test was used. Receiver-operating characteristic curve (ROC) analyses were performed to assess the diagnostic performance of ultrasound and SWV on VTIQ in differentiating benign from malignant thyroid nodules. Areas under the receiver-operating characteristic (ROC) curves $(\mathrm{Az})$ were calculated and compared using the $z$ test. ROC analyses were also used to find out the best diagnostic cut-off value for each VTIQ SWV parameter, and the corresponding sensitivity, specificity, accuracy, PPV, NPV, and accuracy were calculated for this best diagnostic cut-off. The comparisons of sensitivity, specificity, PPV, NPV, and accuracy for differentiating malignant from benign thyroid nodules between ultrasound and VTIQ or between nodules with different size were done by $\chi^{2}$ statistics. The results were considered significant at $P<0.05$. The statistical analyses were performed by using the SPSS 17.0 software package (SPSS Inc, Chicago, IL, United States).

\section{Results}

\section{Histopathologic diagnosis}

There were 113 benign nodules and 93 malignant nodules. Among the benign lesions, there were 47 (68.1\%, 47/113) nodular goiters, $15(21.7 \%, 15 / 113)$ Hashimoto's nodules, $7(10.2 \%, 7 / 113)$ adenomas. Among the malignant lesions, there were $91(98.9 \%, 91 / 93)$ papillary thyroid carcinomas and $1(1.1 \%, 1 / 93)$ undifferentiated thyroid carcinoma.

\section{SWV on VTIQ}

The various SWV values in nodules with different pathological types are showed in the Table 1 . In addition to these, there was one undifferentiated thyroid carcinoma, whose SWV max was $7.21 \mathrm{~m} / \mathrm{s}$, SWV min was $3.35 \mathrm{~m} / \mathrm{s}$, SWV mean was $5.44 \mathrm{~m} / \mathrm{s}$, and SWV median was $5.30 \mathrm{~m} / \mathrm{s}$ respectively.

As for the comparison of SWV value between benign and malignant thyroid nodules, the mean of VTIQ SWV values (including SWV max, SWV min, SWV mean and SWV median) were all significantly different with higher values in malignant nodules: SWV max: $4.07 \pm 1.77 \mathrm{~m} /$ $\mathrm{s}$ (range from 1.93 to $10.0 \mathrm{~m} / \mathrm{s}$ ) vs $3.22 \pm 1.06 \mathrm{~m} / \mathrm{s}$ (range from 2.12 to $6.42 \mathrm{~m} / \mathrm{s})(P<0.001)$; SWV min: $2.97 \pm$ $0.69 \mathrm{~m} / \mathrm{s}$ (range from 1.48 to $4.16 \mathrm{~m} / \mathrm{s}$ ) vs $2.42 \pm 0.73$ 


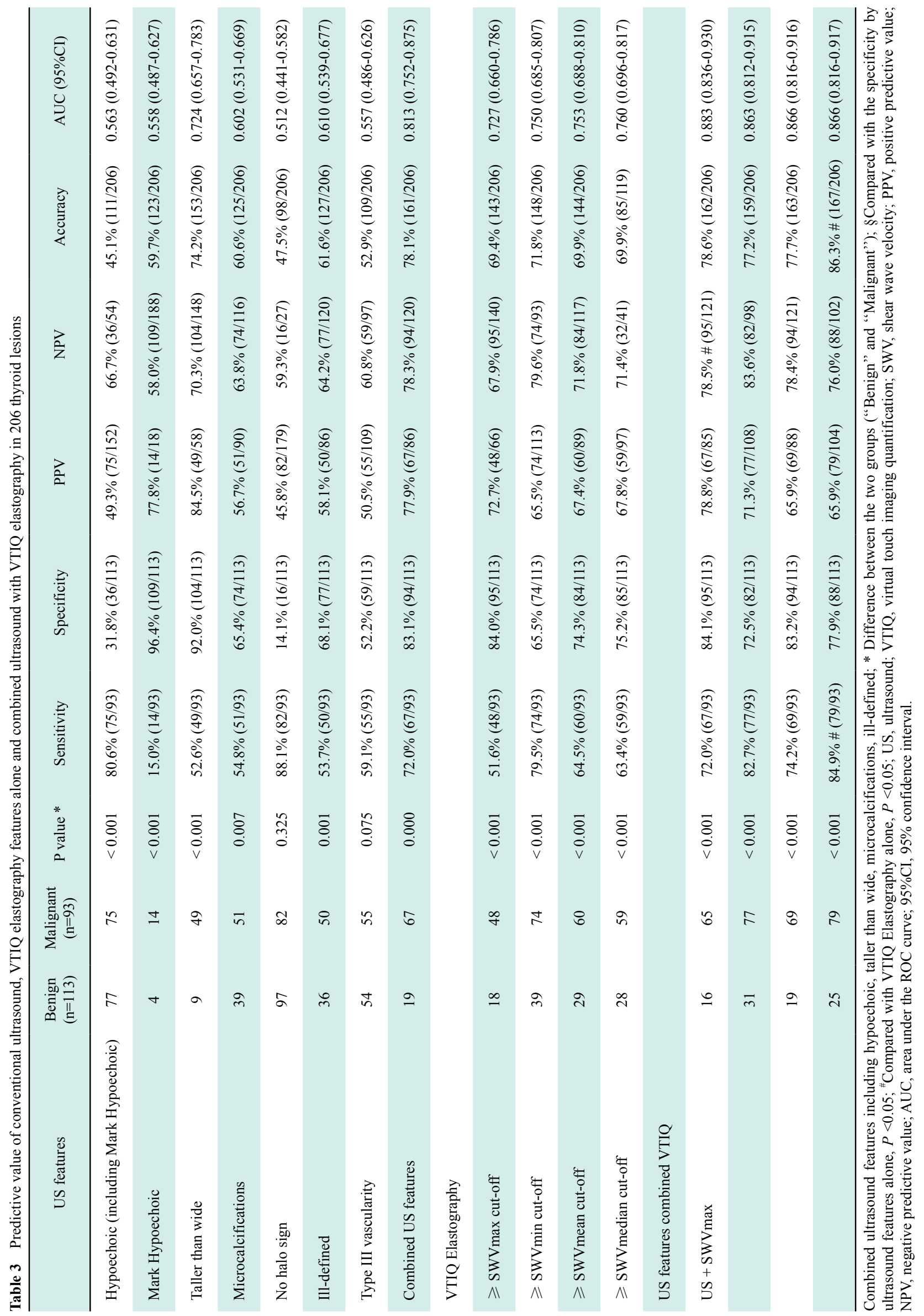


Zhang et al. SWV for differential diagnosis of thyroid nodules

Table 4 Predictive performance of SWV median value for differentiating malignant from benign thyroid nodules in terms of different nodule sizes

\begin{tabular}{|c|c|c|c|c|c|c|c|c|c|}
\hline Nodule Size & $\begin{array}{c}\text { Benign } \\
(n=113)\end{array}$ & $\begin{array}{c}\text { Malignant } \\
(n=93)\end{array}$ & Sensitivity & Specificity & PPV & NPV & Accuracy & $P$ value ${ }^{\#}$ & AUC $(95 \% \mathrm{CI})$ \\
\hline$\leqslant 1.0 \mathrm{~cm}$ & 58 & 71 & $38.0 \%(27 / 71)$ & $84.4 \%(49 / 58)$ & $75.0 \%(27 / 36)$ & $52.6 \%{ }^{* *}(49 / 93)$ & $60.3 \%(76 / 126)$ & $<0.001$ & $0.711(0.624-0.787)^{*}$ \\
\hline $\mathrm{SWV} \geqslant 3.01 \mathrm{~m} / \mathrm{s}$ & 9 & 27 & & & & & & & \\
\hline $\mathrm{SWV}<3.01 \mathrm{~m} / \mathrm{s}$ & 49 & 44 & & & & & & & \\
\hline$>1.0 \mathrm{~cm}$ & 55 & 22 & $72.7 \%(16 / 22)$ & $85.4 \%(45 / 55)$ & $61.5 \%(16 / 26)$ & $88.2 \%(45 / 51)$ & $79.2 \%(61 / 77)$ & $<0.001$ & $0.862(0.764-0.930)$ \\
\hline $\mathrm{SWV} \geqslant 3.13 \mathrm{~m} / \mathrm{s}$ & 10 & 16 & & & & & & & \\
\hline $\mathrm{SWV}<3.13 \mathrm{~m} / \mathrm{s}$ & 45 & 6 & & & & & & & \\
\hline
\end{tabular}

" Compared with AUC of SWV in nodule $>1.0 \mathrm{~cm}, P<0.05 ;{ }^{* *}$ Compared with the predictive performance in nodule $>1.0 \mathrm{~cm}, P<0.05 ;{ }^{\#}$ Difference between the two groups ("Benign" and "Malignant"). SWV, shear wave velocity; PPV, positive predictive value; NPV, negative predictive value; AUC, area under the ROC curve; $95 \% \mathrm{CI}, 95 \%$ confidence interval.

Table 5 Predictive value of conventional ultrasound in 93 malignant thyroid lesions

\begin{tabular}{|c|c|c|c|c|c|c|c|c|c|}
\hline $\begin{array}{l}\text { Ultrasound } \\
\text { features }\end{array}$ & $\begin{array}{l}\text { Lymphatic } \\
\text { metastasis } \\
(n=24)\end{array}$ & $\begin{array}{c}\text { No lymphatic } \\
\text { metastasis } \\
(n=69)\end{array}$ & $P$ value ${ }^{\#}$ & Sensitivity & Specificity & PPV & NPV & Accuracy & AUC $(95 \% \mathrm{CI})$ \\
\hline Halo sign & 7 & 4 & 0.026 & $29.1 \%(7 / 24)$ & $94.2 \%(65 / 69)$ & $63.6 \%(7 / 11)$ & $79.3 \%(65 / 82)$ & $77.4 \%(72 / 93)$ & $0.617(0.510-0.716)$ \\
\hline Microcalcification & 18 & 33 & 0.016 & $75.0 \%(18 / 24)$ & $51.2 \%(36 / 69)$ & $35.3 \%(18 / 51)$ & $85.7 \%(36 / 42)$ & $58.0 \%(54 / 93)$ & $0.634(0.530-0.733)$ \\
\hline
\end{tabular}

"Difference between the two groups of malignant nodules ("lymphatic metastasis" and "No lymphatic metastasis"). PPV, positive predictive value; NPV, negative predictive value; AUC, area under the ROC curve; $95 \%$ CI, $95 \%$ confidence interval.

$\mathrm{m} / \mathrm{s}$ (range from 1.26 to $4.22 \mathrm{~m} / \mathrm{s})(P<0.001)$; SWV mean: $3.46 \pm 0.88 \mathrm{~m} / \mathrm{s}$ (range from 1.74 to $7.08 \mathrm{~m} / \mathrm{s}$ ) vs $2.86 \pm 0.881 \mathrm{~m} / \mathrm{s}$ (range from 1.83 to $4.56 \mathrm{~m} / \mathrm{s})(P<$ 0.001 ); SWV median: $3.42 \pm 0.86 \mathrm{~m} / \mathrm{s}$ (range from 1.79 to $7.42 \mathrm{~m} / \mathrm{s}$ ) vs $2.84 \pm 0.81 \mathrm{~m} / \mathrm{s}$ (range from 1.78 to $4.68 \mathrm{~m} / \mathrm{s}$ ) $(P<0.001)$ (Table 2).

\section{Diagnostic performance of conventional ultrasound and VTIQ}

The diagnostic performances of the main features of conventional ultrasound in diagnosing malignancy were summarized in Table 3. ROC curve analyses showed that the AUC for combined ultrasound features was 0.813 (95\%CI: 0.752-0.875). The sensitivity, specificity, PPV, NPV, and accuracy of combined statistically significant ultrasound features (including hypoechoic, marked hypoechoic, taller than wide, no halo sign, and illdefined) were $72.0 \%, 83.1 \%, 77.9 \%, 78.3 \%$, and $78.1 \%$ respectively.

The diagnostic performances of each SWV value on VTIQ for predicting malignancy were summarized in Table 2 and Table 3. Among the four SWV parameters, the AUC of SWV median was the largest one, but there were no significant differences compared with the other three parameters (all $P>0.05$ ). The sensitivity, specificity, PPV, NPV, and accuracy of SWV mean were $63.4 \%, 75.2 \%, 67.8 \%, 71.4 \%$, and $69.9 \%$ respectively.

After combined VTIQ (including SWVmax, SWVmin, SWVmean and SWVmedian) with conventional ultrasound, the specificity, NPV, and accuracy all increased compared with VTIQ only, and significant differences were found in the following conditions: when conbined ultrasound with SWV median, the $p$ value of specificity was $<0.05$; When combined ultrasound with SWV max, the p value of NPV was $<0.05$; When combined ultrasound with SWV median, the $p$ value of accuracy was $<0.05$. (Table 3 ).

\section{Diagnostic performance of VTIQ in different nodule sizes}

The SWV median was selected to evaluate the diagnostic performance of VTIQ in thyroid nodules with different sizes since SWV median had the largest AUC of ROC in diagnosing malignant thyroid lesions. For nodules with largest diameter $\leqslant 1.0 \mathrm{~cm}$, AUC was 0.711 (95\%CI: $0.624-0.787$ ) with a cut-off SWV value of 3.01 $\mathrm{m} / \mathrm{s}$. For nodules with largest diameter $>1.0 \mathrm{~cm}$, AUC was 0.862 (95\%CI: 0.764-0.930) with a cut-off SWV value of $3.13 \mathrm{~m} / \mathrm{s}$. The sensitivity, specificity, NPV and, accuracy of SWV median in differentiating thyroid nodules > $1.0 \mathrm{~cm}$ were higher than those in nodules $\leqslant 1.0 \mathrm{~cm}$ (all $P>0.05$ ), except for PPV. Furthermore, the AUC and NPV for nodules $>1.0 \mathrm{~cm}$ were higher than those for nodules $\leqslant 1.0 \mathrm{~cm}$ significantly (both $P<0.05$ ) (Table 4).

\section{Diagnostic performance of conventional ultrasound and VTIQ in lymphatic metastasis}

The diagnostic performances of ultrasound features 
and each SWV value on VTIQ for predicting lymphatic metastasis of malignant thyroid nodules were summarized in Table 5. Conventional ultrasound features showed that halo sign and microcalcifications were significant for predicting lymphatic metastasis (all $P>0.05$ ), and the AUC was 0.617 (95\% CI: 0.510-0.716) for halo sign and 0.634 (95\% CI: 0.530-0.733) for macrocalcifications, respectively. There were no significant difference for SWV values on VTIQ (all $P>0.05$ ).

\section{Discussion}

A consensus has been reached that elastography is able to show the inside mechanical properties of lesion, and it is a complement tool of conventional ultrasound in differentiating malignant thyroid lesion from benign ones [9]. A variety of elastography approaches such as supersonic shear imaging (SSI), ARFI imaging as well as strain elastography have been studied in recent years. It is important to find out the more accurate and less operator dependent imaging techniques. VTIQ is a new elastography technique, as opposed to free hand elastography, which employs automatically generated ARFI to induce shear waves within the targeted area. It is a quantitative method and less operator dependent compared with strain elastography.

There were several studies about VTIQ technique on the other superficial organs: testicular lesions (mean SWV for benign lesions was $2.37 \mathrm{~m} / \mathrm{s}$, for germ cell tumors was $1.94 \mathrm{~m} / \mathrm{s}$ and for seminoma was $2.42 \mathrm{~m} / \mathrm{s}$ ), salivary (mean SWV for benign lesions was $4.24 \mathrm{~m} / \mathrm{s}$, for malignant lesions was $6.52 \mathrm{~m} / \mathrm{s}, P<0.05$ ), and breast masses (mean SWV for malignant lesions versus benign lesions: $7.73 \pm$ $1.02 \mathrm{~m} / \mathrm{s}$ versus $4.46 \pm 1.87 \mathrm{~m} / \mathrm{s} ; P<0.0001)$. Unlikely the above studies which only analyzed the SWV max values of the lesions, four SWV values on VTIQ (including SWV max, SWV min, SWV mean and SWV median) were evaluated in our study [10]. The results found that the all SWVs on VTIQ in malignant lesions were significantly higher than those in benign ones.

SSI is also a 2D-SWE technique, and it also depends less on the individual operator and is more reproducible and quantitative. Several reports have been released about SSI for the quantitative evaluation of thyroid nodules [11]. The results showed that quantitative parameters of this elastography technique were significantly different between malignant and benign nodules, with the sensitivities ranged from $43.6 \%-97 \%$ and the specificities ranged from $71.1 \%-97 \%$. In this study of VTIQ, the abilities to predict malignancy of each SWV parameters were slightly lower. As for the diagnostic performance of SWE combined conventional ultrasound, Park et al. found that combining gray-scale ultrasound findings and each SWE elasticity indices showed elevated sensitivity and elevated AUC for predicting malignancy, compared with the use of grayscale ultrasound only [12]. Similar findings were found in our study. After combined VTIQ with conventional ultrasound, the sensitivity, specificity, PPV, NPV, and accuracy were all increased compared with conventional ultrasound only, and the sensitivity and NPV were increased significantly compared with VTIQ only. Therefore, VTIQ could be used as an adjunctive tool for diagnosis of malignancy in addition to conventional ultrasound in clinical practice.

Although SSI and VTIQ are both 2D SWE, VTIQ could present not only the elasticity information of lesions, but also the quality of elasticity [13]. The quality map can be used to confirm that shear wave formation was adequate and to identify regions of the shear wave image where velocity estimation may be less accurate because of poor signal quality [14]. Poor quality can be caused by lack of shear wave generation in cystic lesions or in malignant lesions [15]. In this study, for example, one of the 14 lesions with poor quality of SWE examination received surgery at last and was proved to be malignant. However, the SWV median of the lesion was only 2.14 $\mathrm{m} / \mathrm{s}$ suggestive of a benign lesion. On gray scale image, this nodule showed some macrocalcifications. The macrocalcifications might reduce the acoustic radiation force propagating through the nodule, which resulted in insufficient shear wave generated, and then inadequate measurement was obtained. Barr et al. also described this phenomenon in their study [2]. In some breast cancers, it can be difficult to adequately visualize and measure SWV because of high attenuation [16]. In such cases, the estimate of SWV may be less than the best diagnostic cut-off value because of the poor quality of the data. The quality map can be used to indicate where the shear wave generation was insufficient and the measurement in this area might lead to misdiagnosis [17]. The quality map is useful to reduce false negative cases and to improve the sensitivity of VTIQ.

The present study showed relatively inferior diagnostic performance for nodules $\leqslant 1.0 \mathrm{~cm}$ with a "softer" cut-off value of $3.01 \mathrm{~m} / \mathrm{s}$ (the cut-off value in nodules $>1.0 \mathrm{~cm}$ was $3.13 \mathrm{~m} / \mathrm{s}$ ). These findings were similar with previous studies. Liu et al. found that in the group of nodules $\leqslant 10 \mathrm{~mm}$, the AUC was 0.730 , while it was 0.883 in nodules sized $11-30 \mathrm{~mm}$ and 0.821 in nodules $>30 \mathrm{~mm}$ [18]. The optimal cut-off value in nodules $\leqslant 10 \mathrm{~mm}$ was only $22.8 \mathrm{kPa}$, as opposed to at least $42.7 \mathrm{kPa}$ in bigger nodules. The reason for these differences might be that nodules $\leqslant 1.0 \mathrm{~cm}$ does not arise obvious changes in morphology, which making nodules $\leqslant 1.0 \mathrm{~cm}$ "softer" than bigger ones. Likewise, 
the nodules $\leqslant 1.0 \mathrm{~cm}$ often have some atypical conventional ultrasound features $[19,20]$.

There were several limitations in this study. Firstly, selection bias may exist because most patients included in this study were suspicious for malignancy and scheduled for FNA and surgeries. As a result of this, the ratio of malignancy was higher than that in general population. Secondly, VTIQ was performed by one radiologist and the inter- and intra-observer variabilities were not able to assess in our research. However, another study already showed that VTIQ had good inter-examiner agreement with a strong positive correlation $(\gamma=0.93)$ [21]. Thirdly, in the malignant lesions, there were $59(98.3 \%)$ papillary thyroid carcinomas and $1(1.7 \%)$ undifferentiated thyroid carcinoma. No follicular carcinoma was included. The usefulness of VTIQ for assessing other pathological types of thyroid carcinoma is unknown. Fourthly, VTIQ is capable of four discrete display modes: Velocity, Quality, Travel Time, and Displacement. The "Travel Time" mode and "Displacement" mode were not evaluated in this preliminary study. Further studies are necessary to evaluate the value of those modes in the future.

In conclusion, VTIQ provides quantitative stiffness information of thyroid lesions, which is useful for differentiating between benign and malignant thyroid nodules. The diagnostic performance of combined VTIQ and conventional US is better than that of using VTIQ or US alone. The evaluation of small thyroid lesions remains a difficult problem.

\section{Acknowledgments}

This work was supported in part by grant SHDC12014229 from Shanghai Hospital Development Center; grant 14441900900 from Science and Technology Commission of Shanghai Municipality; grant 20144Y0148 from Shanghai Municipal Commission of Health and Family Planning; grant 81401417 and 81472579 from the National Natural Science Foundation of China.

\section{Conflicts of Interest}

All the authors certify that there is no actual or potential conflict of interests in this article.

\section{References}

[1] Balleyguier C, Canale S, Ben Hassen W, Vielh P, Bayou EH, Mathieu $\mathrm{MC}$, et al. Breast elasticity: principles, technique, results: an update and overview of commercially available software. Eur J Radiol 2013; 82: 427-34.

[2] Barr RG, Zhang Z. Shear-wave elastography of the breast: value of a quality measure and comparison with strain elastography. Radiology 2015; 275: 45-53.

[3] Bhatia KS, Tong CS, Cho CC, Yuen EH, Lee YY, Ahuja AT. Shear wave elastography of thyroid nodules in routine clinical practice: preliminary observations and utility for detecting malignancy. Eur Radiol 2012; 22: 2397-406.
[4] Cappelli C, Pirola I, Gandossi E, Agosti B, Cimino E, Casella C, et al. Real-time elastography: a useful tool for predicting malignancy in thyroid nodules with nondiagnostic cytologic findings. $J$ Ultrasound Med 2012; 31: 1777-82.

[5] Golatta M, Schweitzer-Martin M, Harcos A, Schott S, Gomez $\mathrm{C}$, Stieber A, et al. Evaluation of virtual touch tissue imaging quantification, a new shear wave velocity imaging method, for breast lesion assessment by ultrasound. Biomed Res Int 2014; 2014: 960262.

[6] Kim H, Kim JA, Son EJ, Youk JH. Quantitative assessment of shear-wave ultrasound elastography in thyroid nodules: diagnostic performance for predicting malignancy. Eur Radiol 2013; 23: 2532-7.

[7] Liu B, Liang J, Zheng Y, Xie X, Huang G, Zhou L, Wang W, Lu M. Two-dimensional shear wave elastography as promising diagnostic tool for predicting malignant thyroid nodules: a prospective singlecentre experience. Eur Radiol 2015; 25: 624-34.

[8] Matsuzuka T, Suzuki M, Saijo S, Ikeda M, Matsui T, Nomoto Y, et al. Stiffness of salivary gland and tumor measured by new ultrasonic techniques: Virtual touch quantification and IQ. Auris Nasus Larynx 2015; 42: 128-33

[9] Moon HJ, Sung JM, Kim EK, Yoon JH, Youk JH, Kwak JY. Diagnostic performance of gray-scale US and elastography in solid thyroid nodules. Radiology 2012; 262: 1002-13.

[10] Moon HJ, Son E, Kim EK, Yoon JH, Kwak JY. The diagnostic values of ultrasound and ultrasound-guided fine needle aspiration in subcentimeter-sized thyroid nodules. Ann Surg Oncol 2012; 19: 52-9.

[11] Park AY, Son EJ, Han K, Youk JH, Kim JA, Park CS. Shear wave elastography of thyroid nodules for the prediction of malignancy in a large scale study. Eur J Radiol 2015; 84: 407-412.

[12] Ragazzoni F, Deandrea M, Mormile A, Ramunni MJ, Garino F, Magliona G, et al. High diagnostic accuracy and interobserver reliability of real-time elastography in the evaluation of thyroid nodules. Ultrasound Med Biol 2012; 38: 1154-62.

[13] Russ G, Royer B, Bigorgne C, Rouxel A, Bienvenu-Perrard M, Leenhardt L. Prospective evaluation of thyroid imaging reporting and data system on 4550 nodules with and without elastography. Eur $J$ Endocrinol 2013; 168: 649-55.

[14] Shiina T, Nightingale KR, Palmeri ML, Hall TJ, Bamber JC, Barr $\mathrm{RG}$, et al. WFUMB guidelines and recommendations for clinical use of ultrasound elastography: Part 1: basic principles and terminology. Ultrasound Med Biol 2015; 41: 1126-47.

[15] Sebag F, Vaillant-Lombard J, Berbis J, Griset V, Henry JF, Petit P, et al. Shear wave elastography: a new ultrasound imaging mode for the differential diagnosis of benign and malignant thyroid nodules. J Clin Endocrinol Metab 2010; 95: 5281-8.

[16] Tanter M, Bercoff J, Athanasiou A, Deffieux T, Gennisson JL, Montaldo G, et al. Quantitative assessment of breast lesion viscoelasticity: initial clinical results using supersonic shear imaging. Ultrasound Med Biol 2008; 34: 1373-86.

[17] Szczepanek-Parulska E, Woli ń ski K, Stangierski A, Gurgul E, Biczysko M, Majewski P, et al. Comparison of diagnostic value of conventional ultrasonography and shear wave elastography in the prediction of thyroid lesions malignancy. PLoS One 2013; 8: e81532.

[18] Trottmann M, Marcon J, D'Anastasi M, Karl A, Stief CG, Reiser M, et al. The role of VTIQ as a new tissue strain analytics measurement technique in testicular lesions. Clin Hemorheol Microcirc 2014; 58: 195-209.

[19] Trimboli P, Guglielmi R, Monti S, Misischi I, Graziano F, Nasrollah $\mathrm{N}$, et al. Ultrasound sensitivity for thyroid malignancy is increased by real-time elastography: a prospective multicenter study. J Clin Endocrinol Metab. 2012 Dec; 97(12): 4524-30.

[20] Veyrieres JB, Albarel F, Lombard JV, Berbis J, Sebag F, Oliver C, et al. A threshold value in Shear Wave elastography to rule out malignant thyroid nodules: a reality? Eur J Radiol 2012; 81: 3965-72.

[21] Zhang YF, He Y, Xu HX, Xu XH, Liu C, Guo LH, et al. Virtual touch tissue imaging on acoustic radiation force impulse elastography: a new technique for differential diagnosis between benign and malignant thyroid nodules. J Ultrasound Med 2014; 33: 585-95. 